\title{
HIGHLIGHTS
}

DIABETES

\section{Nocturnal hypoglycemia is frequent in patients with type 1 diabetes mellitus}

Episodes of nocturnal hypoglycemia are frequent and can be prolonged in patients with type 1 diabetes mellitus (T1DM), Beck and colleagues have found.

"Hypoglycemia while sleeping is an extremely important management problem in T1DM," explains lead researcher, Roy Beck (Jaeb Centre for Health Research, Tampa, USA), "as it can lead to seizures or coma and in rare cases even death."

To assess the frequency of nocturnal hypoglycemia in patients with T1DM, Beck et al. analyzed overnight glucose data previously obtained in a randomized trial of continuous glucose monitoring. 36,467 nights of data from 176 patients aged 8-72 years were included in the analysis; a hypoglycemic episode was defined as two consecutive continuous glucose monitoring readings $\leq 3.3 \mathrm{mmol} / 1$ in a $20 \mathrm{~min}$ period.

Hypoglycemia occurred during $8.5 \%$ of the study nights-equivalent to approximately two episodes per month per patient. The median duration of hypoglycemia was $53 \mathrm{~min}$, with $11 \%$ of events lasting $\geq 3 \mathrm{~h}$. The researchers found that high hypoglycemia risk was associated with low baseline $\mathrm{HbA}_{1 \mathrm{c}}$ levels.

Once nocturnal hypoglycemia has been identified in a patient, Beck points out, overnight insulin delivery and the type of bedtime snack eaten could be modified to minimize the occurrence of these events.

Ultimately, this study "reinforces the need for an artificial pancreas in which the insulin delivery from the pump is adjusted based on the glucose readings from the continuous glucose monitoring device," concludes Beck.

\section{Claire Greenhill}

Original article Juvenile Diabetes Research Foundation Continuous Glucose Monitoring Study Group. Prolonged nocturnal hypoglycemia is common during 12 months of continuous glucose monitoring in children and adults with type 1 diabetes. Diabetes Care 33, 1004-1008 (2010) 